\title{
Migration and Transformation of Persistent Organic Pollutants (POPs) in the Atmosphere \\ He Zhang
}

School of Environmental Science and Engineering, North China Electric Power University, Baoding 071000, China.

summerlight@qq.com

Keywords: persistent organic pollutants, POPs, migration, transformation

\begin{abstract}
Persistent Organic Pollutants (POPs) are huge groups of chemicals that are associated with a variety of human health problems. They have a long residual, bioaccumulation, semi-volatile and high toxicity and they can migrate through various environmental media (air, water, soil, organisms, etc.) over long distances, which means that they have a seriously harmful effect on human's health and the environment. Learning the migration and transformation of POPs in the environment is of great importance to control POPs pollution.
\end{abstract}

\section{Introduction}

Persistent organic contaminants (POPs) refer to some organic chemical contaminants which have stable chemical properties and are hard to break down in the environment. They can accumulate into organism through the food chain and have high toxicity and semi-volatile. Because POPs are semi-volatile and difficult to degrade, atmospheric circulation can make it transport for a long-range in the atmosphere, thus POPs are distributed in every corner of the world. This article will focus on the source of atmospheric POPs, and the migration, transformation of POPs in the atmosphere.

\section{Types of POPs}

127 countries and territories pass through the "Stockholm Convention" in 2001, and decided to prohibit or restrict the use of 12 kinds of POPs divided into three categories around the world, their specific names are listed in Table 1:

Table 1 Name and classification of POPs

\begin{tabular}{ll}
\hline \multicolumn{1}{c}{ Source } & \multicolumn{1}{c}{ POPs name } \\
\hline $\begin{array}{l}\text { Organochlorine } \\
\text { pesticide }\end{array}$ & $\begin{array}{l}\text { DDT, chlordane, mirex, aldrin, dieldrin, endrin, } \\
\text { heptachlor, toxaphene }\end{array}$ \\
Industrial chemicals & hexachlorobenzene, polychlorinated biphenyls \\
Byproducts & dioxins, furans
\end{tabular}

Since the "Stockholm Convention" come into force in 2004, each contracting states strictly control and reduce the above-mentioned 12 kinds of POPs (called "dirty dozen"), but in recent years, many new organic pollutants continue to be discovered from a variety of environmental media, such as brominated flame retardants, perfluorinated compounds and so on. Conference of the Parties of the Stockholm Convention at its fourth session(COP-4) in 2009.5, which held in Geneva, Switzerland, decided to put nine kinds of new chemical substances in the Convention, they are: perfluorooctane sulfonic acid and its salts, perfluorooctane sulfonyl fluoride, commercial pentabromodiphenyl Union diphenyl ether, commercial octabromodiphenyl ether, chlordecone, lindane, pentachlorobenzene, $\alpha$ - BHC, $\beta$ - BHC and hexabromodiphenyl. 


\section{The sources of atmospheric POPs}

Atmospheric POPs have several sources.

Volatilization of pesticides. Loss and volatilization during the application of pesticides, and pesticide residues volatilize from plants and soil surface after application of pesticides may make some POPs volatilize into the air.

Industrial pollution. Brominated flame retardants (BFRs), which arewidely used in furniture, plastics, electronics and other devices due to good flame retardant properties, can enter the environment by using, disposal and other processes. Polybrominated diphenyl ethers(PBDEs) is currently the most widely used BFRs. Tetrabromobisphenol A(TBBPA) is a reactive flame retardant, the average annual demand of global is about 120,000 tons, which is the largest production and use of BFRs currently. Besides, there are perfluorinated compounds (PFCs) widely used in the textile, paper, leather and other industries, because they are oleophobic and hydrophobic.

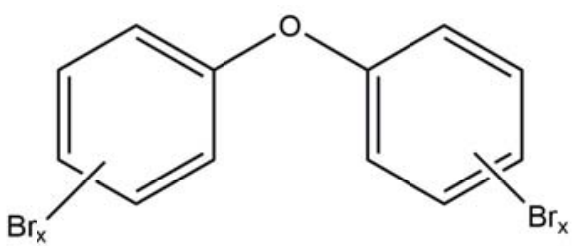

Polybrominated diphenyl ethers(PBDEs)

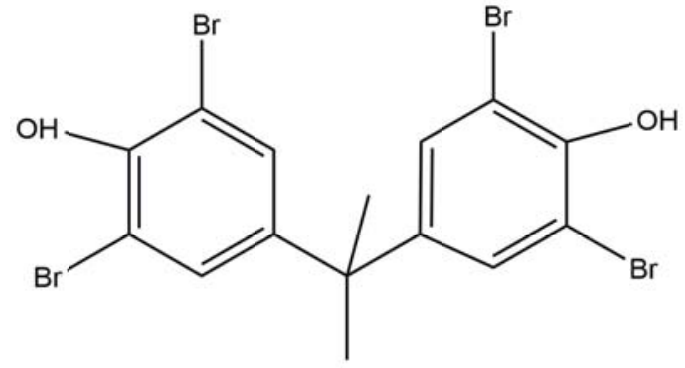

TetrabromobisphenolA(TBBPA)

Figure 1 Molecular structure of PBDEs and TBBPA

Motor vehicle emissions. A study found that the concentration of halogen pollutants in the atmosphere, decreased along the city to the countryside, and the concentration of extractable organic chlorine $(\mathrm{EOCl})$ in the transportation hub region is much higher than it in sampling points away from the hub. According to this phenomenon, we can get a conclusion that automobile exhaust is one of the sources of organochlorine contaminants in the atmosphere.

Waste incineration. Incomplete combustion of fossil fuels and biomass will produce polycyclic aromatic hydrocarbons (PAHs), and enter the atmosphere in the form of gaseous and particulate.

\section{The migration of POPs in the atmosphere}

The long-range atmospheric transport of POPs. Globally, in summer, the temperature is high, POPs can easily volatilize and migrate in midlatitudes, while at lower temperatures in winter, they are easy to settle. POPs have a series of relatively short distance jump to the high latitudes during the migration, known as the "Grasshopper effect", Waina et al. established a suitable global migration patterns, and the principle of it is similar to the gas chromatography separation principle (distillation effect). Wania think, POPs migrate from lower latitudes to high latitudes through a series of grasshopper form, the power of migration is seasonal variation in temperature. In all latitudes, pollutants exchange between soil and atmosphere, but temperature is high in lower latitudes, the amount of volatile is greater than that of the settlement, the exchange perform as a net evaporation, which is the source of pollutants. While temperature is lower in high altitude, the settlement amount is greater than the amount of volatile, the exchange perform as a net settlement, so the high latitude area is the pollutants sink area. Eventually it leads POPs to migrate from the tropics to the cold regions, and it is why POPs are found in polar and alpine regions, where never used POPs. The global migration model of POPs is shown in Figure 1. 


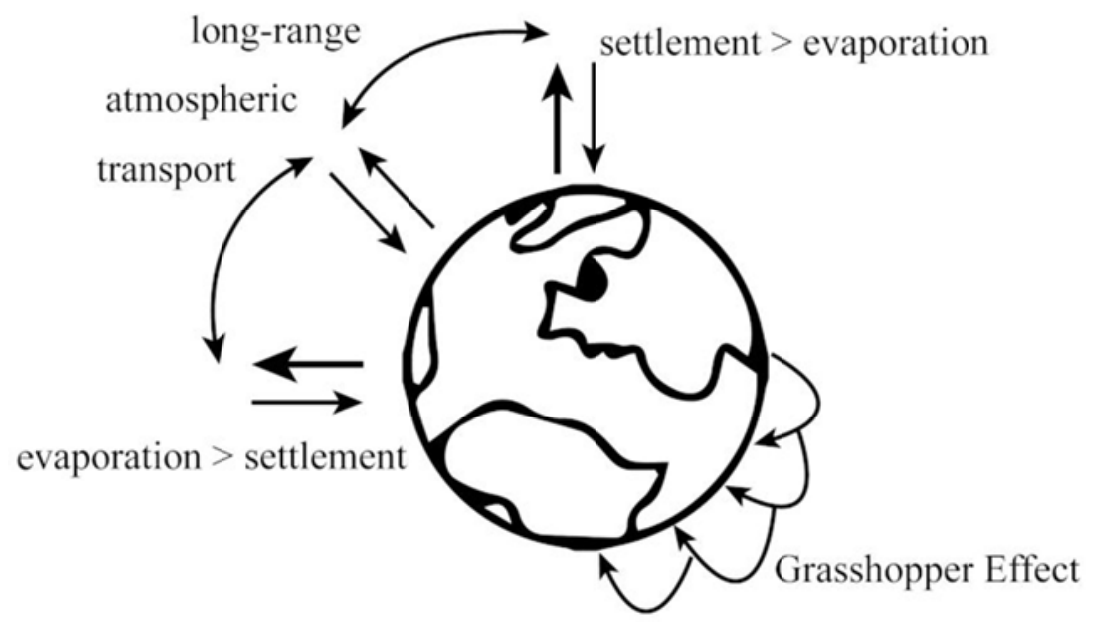

Figure 2 The global migration model of POPs

The settlement of POPs in the atmosphere. Based on the characteristic semi-volatile, POPs exist as gaseous form and the presence of particulate matter in the atmosphere, and produce balanced distribution between the gas phase and particle phase. If the contaminant is adsorbed on the particle phase, it will be easy to eliminate these contaminants in the atmosphere by sedimentation. Sedimentation can be divided into dry deposition and wet deposition. Dry deposition refers to POPs adsorbed on particulate matter fell to the ground surface by gravity, and are adsorbed by surface substances. Wet deposition refers to the atmospheric POPs adsorbed or scoured directly by rain or snow and sink to the ground.

\section{The conversion reaction in the atmosphere of POPs}

Usually, the chemical properties of POPs are very stable, and it is hard to be hydrolyzed, not easy to react with acid and alkali. But under certain conditions, it can be oxidized by strong oxidizing agents, such as hydroxyl radical, and it can absorb ultraviolet light and carry on actinic degradation under the action of the photocatalyst and the photosensitizer.

Take polychlorinated biphenyls (PCBs) for example, in natural environment, elimination of PCBs mainly depends on the photodegradation and microbial degradation effect. Studies have shown that the morethe numbers of chlorine atoms on the benzene ring of PCBs are, the photolysis reaction is more rapid. Ortho substituted chlorine atom on the benzene ring has greater optical activity, PCBs direct photolysis generate orthodechlorination product that is more dominat. Photodegradation is continuous dechlorination, and mainly for the ortho and para dechlorination, produced dechlorination products and small amounts of derivatized byproducts.

\section{Summary}

Although POPs have been disabled for many years, the world is still facing severe POPs pollution, from the view of the geographical features, there are still a large number of areas produce and use POPs, its POPs concentration in the atmosphere is much higher than regional without using it. The impact on the environment of POPs is not regional, it can affect the whole world through various environmental media, such as atmospheric referred to herein, as well as water, soil and organisms. It's urgent to make more national attention to POPs pollution problem, and initiate treatment early. POPs are highly toxic, possibly causing serious harm on human health and ecological harmony, but it is hard to determine the extent of the impact now. We should do a thorough study of POPs to have a more detailed understanding of it, and learn its detailed migration and transformation mechanisms in different environmental media to avoid it from hurting us. 


\section{Reference}

[1]Zhengkan Liu, Limin Yang, QiuquanWang. Recent Approaches to the analysis of atmospheric persistent organic pollutants,

[2]Qingqing Zhu, XianZhang, Shujun Dong et al. Progress on the gas / particle partitioning of persistentorganic pollutants in atmosphere, Environmental Chemistry, 2014,33(10):1629-1635

[3]Xiaoxu Song,Zuwei Wang, Xiaofang Hu. Research Progress of Persistent Organic Pollutants in Different Media, Northern Environmental, 2012, 24(4):135-139 